\title{
Jinping Neutrino Experiment
}

\author{
Shaomin Chen and Zhe Wang* \\ Tsinghua University, China \\ E-mail:Chenshaomindtsinghua.edu.cn, wangzhe-hepdtsinghua.edu.cn
}

This article presents the highlights of the Jinping Neutrino Experiment. China JinPing underground Laboratory (CJPL) has the lowest cosmic-ray moun flux and reactor neutrino background. It is an ideal site for low background neutrino experiments. Jinping Neutrino Experiment, with target mass about 4 kton, was proposed. Jinping will make a leap in the study of solar neutrino physics, providing a tight constraint of the neutrino oscillation upturn, discovering the carbonnitrogen-oxygen (CNO) neutrinos, and addressing the solar metallicity problem. Jinping can also carry a precise measurement on geo-neutrinos with an unambiguous separation on $\mathrm{U}$ and $\mathrm{Th}$ components, and exclude some geo-neutrino predictions.

38th International Conference on High Energy Physics 3-10 August 2016

Chicago, USA

${ }^{*}$ Speaker. 
Jinping Neutrino Experiment (Jinping) [ [, , [] $]$ is a unique observatory for low-energy neutrino physics, astrophysics and geophysics. China JinPing Laboratory (CJPL) has a number of unparalleled features, thickest overburden, lowest reactor neutrino background, etc., which identify it as the world-best low-energy neutrino laboratory (see Fig. W). The target material of the proposed experiment would be about 4 kilotons of liquid scintillator or slow liquid scintillator, with a fiducial mass of 2 kilotons for neutrino-electron scattering events and 3 kilotons for inverse-beta interaction events.

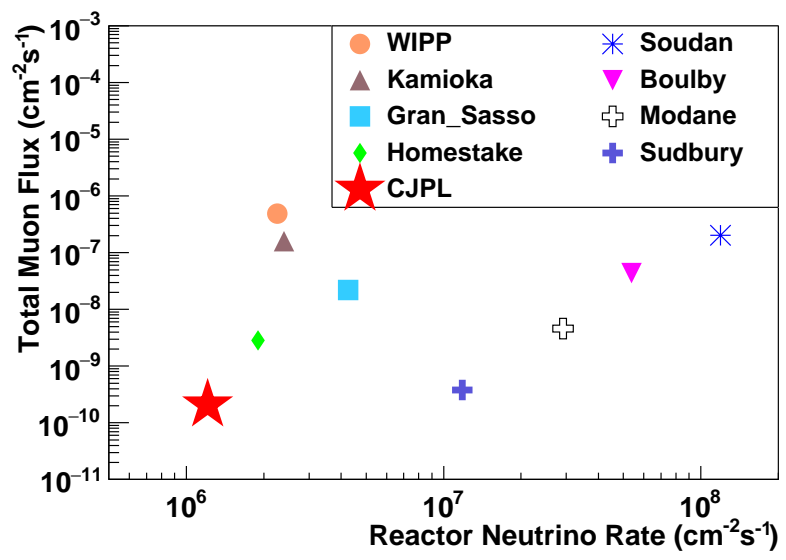

Figure 1: Muon flux vs reactor neutrino background rate for several underground laboratories in the world.

Jinping has a potential to significantly improve the measurements of neutrinos with a few $\mathrm{MeV}$ energy from the interior solar fusion processes, including the components, fluxes and spectra (see Fig. (2). Jinping can precisely measure the transition phase for the solar neutrino oscillation from the vacuum to the matter effect (upturn), providing a critical test for the Mikheyev-SmirnovWolfenstein (MSW) theory in the high density environment. Jinping can discover solar neutrinos from the carbon-nitrogen-oxygen $(\mathrm{CNO})$ cycle with $10 \%$ precision for the $\mathrm{O}-15$ neutrinos, discovering the energy source for massive stars and shedding light on the metal abundance of the solar core and the homogeneous chemical assumption of the heavy elements. The experimental separation of the high and low metallicity hypotheses with the known neutrino oscillation angles is more than 5 standard deviations.

Jinping can carry a precise measurement on geoneutrinos with an unambiguous separation on $\mathrm{U}$ and Th cascade decays from the dominant crustal anti-electron neutrinos. The estimated event rates of $28 \mathrm{U}$ and $7 \mathrm{Th}$ geoneutrino events/year/kton will be significantly above the expected $<6$ reactor neutrino events/year/kton of the signal region. The ratio of U/Th can be determined to $10 \%$. We expect that the measurement from Jinping together with the Borexino and KamLAND results can give an extrapolation of the flux for the desired mantle neutrinos (see Fig. [1) and reveal the mystery of the engine driving Earth's continental growth, mountain movement, and distribution of heat producing elements. 


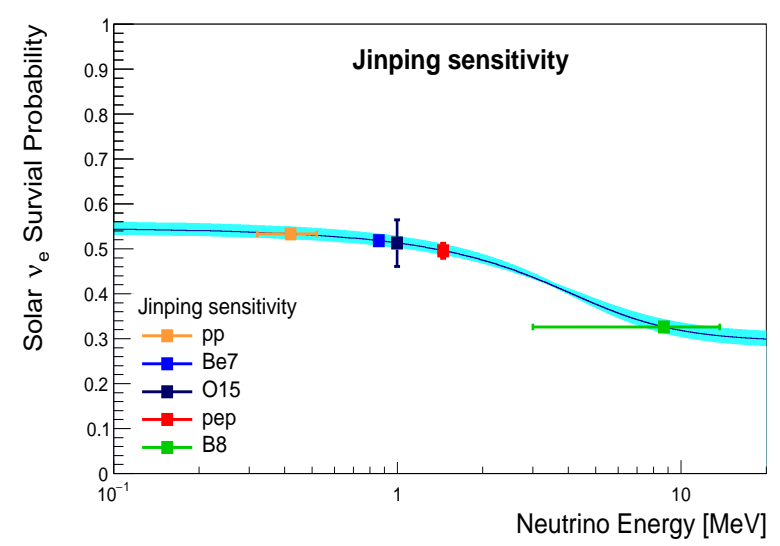

Figure 2: The transition of oscillation probability from the vacuum to matter effect as a function of neutrino energy. The central line is for the theoretical prediction, while the shaded area is obtained by marginalizing $\theta_{12}, \theta_{13}$, and $\Delta m_{12}^{2}$ with the present experimental uncertainties. We assume a 2,000-ton target mass, 1,500day exposure, a resolution of $500 \mathrm{PE} / \mathrm{MeV}$, and the low metallicity hypothesis, and the five points with error bars are the simulation results for $p p,{ }^{7} \mathrm{Be}$, pep, ${ }^{15} \mathrm{O}$ and ${ }^{8} \mathrm{~B}$, in which the central values are set to the true ones, and the y-error bars include both statistical and systematic uncertainties and the $\mathrm{x}$-error bars correspond to the range of energy measurement, while the ${ }^{15} \mathrm{O}$-error is omitted for a clear view.

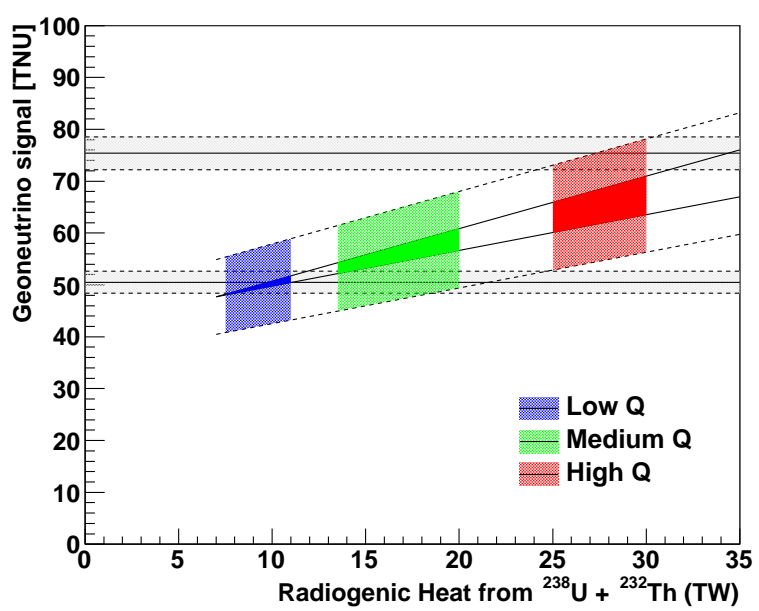

Figure 3: The geo-neutrino sensitivity vs geo-neutrino model predictions at Jinping. The three filled regions in the plot delimit, from the left to the right, low- medium- and high-Q models, respectively. The two horizontal bars are plotted at two possible geo-neutrino flux assumptions with Jinping geo-neutrino measurement sensitivity.

\section{References}

[1] John F. Beacom, Shaomin Chen, Jianping Cheng et al. arXiv:1602.01733 (2016).

[2] http://jinping.hep.tsinghua.edu.cn 